\title{
CIENCIA UNIVERSITARIA PARA DINAMIZAR LA ECONOMÍA LOCAL
}

\author{
Dr.C. Victor Soulary Carracedo \\ Decano de la Facultad de Ciencias Económicas y Empresariales \\ Universidad de Oriente, Cuba \\ vsoulary@uo.edu.cu \\ Dra.C. Marianela González Ortiz \\ Investigadora Principal de la Red Universitaria para la Gestión del Desarrollo Local Sostenible \\ Universidad de Oriente, Cuba \\ nela@uo.edu.cu \\ Dr.C. Ulises Pacheco Feria \\ Director de la Revista Anuario de la Facultad de Ciencias Económicas y Empresariales \\ Universidad de Oriente, Cuba \\ upacheco@uo.edu.cu
}

\section{RESUMEN}

La ciencia universitaria tiene un papel prioritario en la dinamización económica, ya que repercute en la productividad y competitividad de las empresas, y en su destreza para generar riqueza. Las universidades son las instituciones con mayor concentración de recursos avanzados para crear conocimiento; por tanto, se constituyen en uno de los fundamentos del crecimiento económico de las sociedades avanzadas. El artículo reflexiona sobre los antecedentes teóricos y prácticos que avalan la necesidad de evaluar la contribución de la ciencia universitaria a la dinamización económica local. A partir de la revisión crítica del estado del arte se demuestra que evaluar la articulación de los resultados científicos universitarios con el desempeño de los agentes económicos es una herramienta clave en la mejora de la relación simbiótica entre empresas y academias.

PALABRAS CLAVE: Ciencia, universidad, dinamización, economía, desarrollo local, evaluación.

\section{UNIVERSITARY SCIENCE FROM LOCAL ECONOMIC REVITALIZING}

\section{ABSTRACT}

The university science has a priority role in economic revitalizing, since it impact in the productivity and competitiveness of enterprises, and in their skill to generate richness. Universities are the institutions with more advanced resources to create knowledge; therefore they constitute one of the fundaments of economics' growth of advanced societies. This paper intends about the theoretical and practical antecedents that justify the necessity of evaluate the contribution of the 
university's science to the local economic revitalizing. From the critical revision of the state of the art, we argue that assets the articulation of the universities scientific results with the performance of the economic agents is a key tool to improve the symbiotic relationship between enterprises and academies.

KEY WORDS: Science, university, revitalizing, economy, local development, assets.

\section{LA SCIENCE UNIVERSITAIRE POUR AMÉLIORER L'ÉCONOMIE LOCALE}

\section{RÉSUMÉ}

La science universitaire joue un rôle de priorité dans la dynamisation de l'économie puis qu'elle répercute sur la productivité et la concurrence des entreprises, et aussi dans le savoir-faire pour engendrer des richesses. Les universités sont les institutions avec la plus grande concentration de ressources avances pour créer des connaissances, par conséquent, elle devienne en fondement d'accroissement économique des sociétés avances. L'article reflété sur les antécédents théoriques et pratiques qu'avalisent le besoin d'évaluer la contribution de la science universitaire a la dynamisation de l'économie local. À partir de la révision critique de l'état de l'art on démontre qu'évaluer l'articulation des résultats scientifiques universitaires avec l'exercice des agents économiques c'est un outil clef pour améliores la relation symbiotique entre les entreprises et les académies.

MOTS-CLES: Science, université, dynamisation, économie, développement local, évaluation.

\section{1. ¿Resulta imprescindible dinamizar la economía local para conseguir desarrollo?}

Las primeras crisis globales del petróleo, el agotamiento del modelo fordista y la comprensión de la necesidad de modelos de desarrollo flexibles y a escala reducida, provocaron, a finales de los años setenta y principios de los ochenta del siglo XX, la aparición de uno de los términos más en boga en el argot académico contemporáneo: desarrollo local, el cual puede encontrarse referenciado en una voluminosa cantidad de estudios, informes, libros y artículos académicos.

La complejidad del análisis de los procesos de desarrollo local parte desde su conceptualización. Para entender qué es local, primero es menester ubicar el punto de mira. Así, un municipio es local si se mira desde la provincia, pero esta última también lo sería vista desde la nación. Por ello resulta fácil acceder a enfoques múltiples y divergentes sobre el desarrollo de una localidad.

En materia de conceptualización del fenómeno, existen definiciones que afirman que el desarrollo local es el proceso que, mediante el aprovechamiento eficiente de los recursos endógenos existentes, es capaz de estimular el crecimiento económico, crear empleo y mejorar la calidad de vida de la comunidad local (Castillo, Borroeta, Bayón y Cordero, 1994). Así, le asigna la función de proceso reactivador de la economía y su influencia en el desarrollo social, aunque no profundiza en la necesidad de su sostenibilidad

Una visión interesante es la que afirma que el desarrollo local es el proceso endógeno que se opera en pequeñas unidades territoriales sobre la base de sus potencialidades y que es capaz de 
promover el dinamismo económico y la mejora de la calidad de vida de la población, destaca que es una resultante directa de las capacidades de estructuración y movilización de los actores locales para definir y explorar sus prioridades, buscando la competitividad en un contexto de rápidas y profundas transformaciones (Buarque, 1999), con lo cual asigna un peso importante a la dinámica de interrelación entre los sujetos del desarrollo.

Otras conceptualizaciones plantean que el desarrollo local es un proceso de cambio estructural que aumenta el bienestar de la población de una localidad a partir de un crecimiento económico signado por dos características: más equitativo en términos sociales, más sostenible ambientalmente (Vázquez Barquero, 2000å; Alburquerque, 2003). Resalta en estas definiciones la asunción de tres pilares básicos del desarrollo local: crecimiento económico, justicia social y sostenibilidad ambiental.

Un enfoque similar es el que lo propone como un complejo proceso de concertación entre los agentes que interactúan dentro de los límites de un territorio determinado, con el propósito de impulsar un proyecto común que combine la generación de crecimiento económico, equidad, cambio cultural, sustentabilidad ecológica, equilibrio espacial y enfoque de género; con el fin de elevar la calidad de vida y el bienestar de cada ciudadano (Enríquez, 2001); al tiempo que otro punto de vista lo identifica como un proceso localizado de cambio socio-económico continuado que, liderado por los gobiernos locales, integra y coordina la utilización de la riqueza de su potencial de desarrollo con las diferentes corrientes de recursos, para lograr el progreso de la localidad y posibilitar el bienestar del ser humano, en equilibrio con el entorno natural. Estas concepciones no solo reconocen los tres pilares ya mencionados, sino que asignan un papel determinante a la concertación entre actores locales (González Fontes, 2002).

Haciendo énfasis en la dimensión económica del desarrollo local algunos autores declaran que los procesos de desarrollo local se producen gracias a la utilización eficiente del potencial económico local. Incluso reconocen que este tipo de desarrollo pasa primero por el dinamismo de la economía del territorio, pues las iniciativas que se lleven a cabo por las empresas locales deben realizarse con un carácter rentable y eficiente. En igual medida, afirman que es un modo de promover el desarrollo que toma en cuenta el papel de todos los factores necesarios para convertir en dinámicas las potencialidades que pueden identificarse al examinar una unidad socio-territorial delimitada (Vázquez Barquero, 2000b; Orero, 1993; Padilla, Pino y Aguirre, 2004).

Si se analizan las definiciones anteriores, al margen de las diferencias, más de orden semántico que conceptual, se desprenden puntos concurrentes que caracterizan el proceso de desarrollo local: el carácter endógeno, las dimensiones básicas del proceso (económica, ambiental y social), el papel determinante de los actores locales y la importancia clave de la dinamización de la economía.

Lo anterior asigna un peso importante a la economía local como motor impulsor de las transformaciones estructurales, dado que es ella la encargada de generar los recursos materiales y financieros que luego pueden ser utilizados en el desarrollo de otras dimensiones. Tal es así que al definir a la economía local como el conjunto de actividades de producción y servicios de la localidad y asumir que es un potencial que abarca tanto las actividades como los recursos disponibles, sean físicos o sociales, se entiende que su estudio es indispensable para elaborar propuestas de desarrollo local (Méndez, 2005). En el fundamento de este enfoque descansa un principio básico del proceso de desarrollo local: la evolución sostenible de la economía local es el elemento garante de cualquier transformación ulterior. El crecimiento económico y la 
dinamización de la economía es la condición necesaria, aunque no suficiente, para pensar en desarrollo desde la localidad.

En esa dirección, se plantea que una condición necesaria de un proceso de crecimiento económico capaz de sostenerse en el largo plazo es el avance equilibrado y proporcional entre las distintas grandes ramas de la actividad económica en el largo plazo (Nurkse, 1959). Así que el momento inicial en todo proceso de desarrollo es el crecimiento económico como base de la riqueza material, pero no un crecimiento burbuja, incapaz de auto sostenerse en el tiempo; sino uno que sea capaz de hallar actividades económicas que logren, con su propio despliegue, incitar al resto de las actividades de la localidad a crecer.

En ello radican las principales diferencias entre el proceso de crecimiento económico y el proceso de dinamización económica. El primero se caracteriza por el incremento del valor de los bienes y servicios finales producidos por una economía, independientemente de la unidad espacial analizada, en un determinado período, generalmente un año. Lo anterior presupone que si pocas actividades económicas son capaces de hacer crecer el valor total de lo producido, se está en presencia de un proceso de crecimiento económico aun cuando el resto de las actividades no esté creciendo. Por otra parte, el segundo proceso implica no solo el crecimiento de algunas actividades, sino que ese crecimiento sea sostenido en el tiempo, en la mayor parte de las actividades económicas (estén o no enlazadas), y que además se contagie a otros indicadores de actividad económica más allá del valor de los bienes y servicios. O sea, implica que crezcan también otras variables de la economía como el empleo, las inversiones, la rentabilidad, el ahorro, el consumo, entre otras.

Por ende, la dinamización económica local debe ser entendida como el crecimiento sostenido en las actividades económicas del territorio, definidas como priorizadas por las instancias de gobierno, que tienen un impacto significativo en las principales variables de la actividad económica local y en la elevación del nivel de vida de la población.

Existe un número considerable de factores que influyen en la dinamización económica local: el capital invertido, la fuerza de trabajo, así como la ciencia, la tecnología y la innovación puestas al servicio de la producción. Según las teorías económicas del crecimiento, se suele dar tres tipos de respuesta con respecto a las causas del crecimiento económico. La primera dice que la economía crece porque los trabajadores tienen cada vez más instrumentos y medios para realizar sus tareas; es decir, más capital. Para los defensores de esta idea, la clave del crecimiento económico está en la inversión. La segunda respuesta es que los trabajadores con un mayor nivel de conocimientos son más productivos y con la misma cantidad de insumos son capaces de obtener una mayor producción. Entonces, la clave del crecimiento sería la educación, que incrementaría el capital humano o trabajo efectivo. El tercer tipo de respuesta señala que la solución está en obtener mejores formas de combinar los insumos, máquinas superiores y conocimientos más avanzados. Los defensores de esta respuesta afirman que la base del crecimiento económico se encuentra en el progreso tecnológico.

La evidencia empírica y los hechos estilizados parecen apuntar la supremacía teórica y práctica del tercer enfoque, que afirma que no basta con el capital físico acumulado o la mano de obra calificada, sino que se precisa que dicho personal utilice eficientemente los recursos de que dispone sobre la base de la introducción racional y ordenada de los adelantos científicos; lo que conlleva aceptar que la ciencia, la tecnología y la innovación son los factores esenciales en el crecimiento económico, y en la dinamización de la economía. 
De hecho, desde la formulación del modelo neoclásico de crecimiento hasta los más recientes modelos de crecimiento endógeno, se ha tratado de demostrar empíricamente que una vez llegadas las economías al estado estacionario, ni más capital ni más trabajo harán crecer la producción; solo el progreso tecnológico podrá expandir la frontera de posibilidades de producción de la economía (Solow, 1956; Romer, 1986; Lucas, 1988; Rebelo, 1991; Mankiw, Romer y Weil: 1992).

Uno de los modelos teóricos más completos de crecimiento económico es el propuesto por Romer, el cual, establece que la tecnología es un factor clave en el incremento de la producción de bienes y servicios. En materia de tecnología, asigna un peso importante a la generación de ideas como factor impulsor del crecimiento, al considerar que en la creación incesante descansan las potencialidades mayores de desarrollar la tecnología y con ella de reactivar la producción de bienes y servicios. Entiéndase por ideas a aquella parte de la actividad cognitiva humana destinada a la creación científica; o en términos más amplios, a la generación y canalización del conocimiento científico (Romer, 1986).

Otros autores han delimitado el rol de las instituciones en los procesos de crecimiento, e incluso, han particularizado la función clave de aquellas instituciones que generan ideas (universidades y centros científicos) en la consecución de objetivos de crecimiento económico, así como el complejo entramado que relaciona a los centros científicos con la producción (Castro, 2000; Castro, 2004; Acemoglu, Johnson y Robinson, 2005). La anterior línea de pensamiento se asienta en los postulados de North y Thomas, quienes sostienen que los factores que contienen las funciones de producción de los modelos neoclásicos para explicar el crecimiento no son las causas del crecimiento si no que son el crecimiento (North y Thomas, 1973). Según esta visión, la explicación fundamental de las diferencias en el crecimiento son las instituciones.

\section{2. ¿Existe contribución de la ciencia universitaria a la dinamización económica local?}

Con la transición de la sociedad hacia una nueva etapa de desarrollo (la economía basada en el conocimiento), la ciencia, la tecnología y la innovación se han convertido en los factores esenciales en los procesos productivos actuales; asumiendo, de esta manera, un mayor protagonismo en el desarrollo, sea nacional o local. El acelerado desarrollo tecnológico de los últimos años ha convertido al conocimiento en un factor productivo más, de especial interés.

Las actividades de ciencia, tecnología e innovación son desarrolladas por un conjunto de actores entre los que destacan: los Parques Científicos y Tecnológicos, los Centros e Institutos Tecnológicos y los Centros de Empresas e Innovación, pertenecientes al sistema tecnológico de la sociedad; los Organismos Públicos y organizaciones no gubernamentales, pertenecientes al sistema públicoinstitucional; las empresas, pertenecientes al sistema productivo; y las universidades, pertenecientes al sistema científico (Bueno, 2001). Justamente, de la interacción entre los últimos actores depende en buena medida que la ciencia se convierta en un factor productivo de especial importancia.

El papel de las universidades en la dinamización económica local ha sido abordado, en lo fundamental, como un vínculo imprescindible con el sector productivo que debe establecerse a partir de la transferencia de tecnología y de la formación y superación de la fuerza de trabajo que se precisa para emprender endógenamente las iniciativas locales de desarrollo. 
Dado el incesante proceso de innovación y cambio que caracteriza al mundo actual, el desarrollo de nuevas capacidades resulta un elemento crucial, por lo que el desarrollo local exige la movilización y valorización de los recursos humanos como tarea decisiva. La capacitación de los recursos humanos, que depende en lo fundamental de la actividad universitaria, es, por consiguiente, un recurso estratégico y una inversión productiva determinante, y como tal, debe integrar las características específicas de cada territorio, con el fin de diseñar apropiadamente sus contenidos (Vázquez Barquero, 2000a; Arocena, 2002; Alburquerque, 2003).

Otra de las funciones clave de una universidad en una localidad es vincular activamente la ciencia a la producción de bienes y servicios para impulsar el desarrollo local, con lo cual la transferencia tecnológica de la ciencia básica universitaria a las empresas locales es un proceso que garantiza beneficios a las partes involucradas, además de brindarles a las universidades la posibilidad de instituirse como agentes animadores de iniciativas innovadoras de desarrollo local (Alburquerque, 2001).

Una de las fuentes esenciales del crecimiento económico se asienta en la triple hélice que establecen las universidades, las empresas y los gobiernos. Si el conjunto de relaciones funcionales que se establecen entre estos actores socioeconómicos es idóneo, planificado y conducido, las universidades, como creadoras de conocimiento, impactan decisivamente en la elevación de la productividad de las empresas y en los resultados de las economías locales. Incluso, estas hélices pueden ser de variada tipología, en función de la robustez y eficacia de las relaciones establecidas entre estado, industria y academia. Los aspectos más interesantes de este entramado relacional se asientan, primero, en el hecho de que, cuando están bien definidas e intencionadas, permiten que la innovación sea un fruto genuino de la interacción entre los actores y no del mandato político de una institución; y segundo, que estos actores, además de cumplir con sus funciones tradicionales, se nutren de los roles del resto, potenciando sus funciones en el desarrollo local. Este modelo defiende una universidad empresarial, que toma los papeles tradicionales de la industria y el gobierno, que es la institución central para innovar en las regiones (Etzkowitz y Klofsten, 2005).

Las universidades organizan su actividad de ciencia, tecnología e innovación a través de los proyectos científicos. Un proyecto se concibe como la unidad mínima operacional que vincula recursos, actividades y componentes durante un período determinado y con una ubicación definida. Es la búsqueda de una solución inteligente al planteamiento de un problema, que aspira a resolver, entre tantas, una necesidad humana (Sapag, 2000). Esa es justamente la intención de los proyectos científicos universitarios: combinar recursos financieros y materiales con el capital humano que se mueve dentro de los muros de la universidad para obtener soluciones a los problemas de la sociedad.

Lo anterior permite asumir que una línea de investigación universitaria posee orientación dinamizadora cuando está orientada, en lo fundamental, a resolver problemas de la localidad que impiden el proceso de dinamización económica, o a potenciar factores que impulsan dicho proceso, aportando además relevancia científica y visibilidad internacional. Ello debe quedar constatado no solo en los objetivos y líneas de investigación del proyecto, sino, sobre todo, en los resultados obtenidos, que deben ser relevantes en materia científica para la universidad y su prestigio académico, pero también pertinentes en materia económica para el territorio. Si a lo anterior se sumara la aparición de financistas locales o internacionales, interesados en presupuestar las actividades científicas por el aporte que realizan a la solución de problemas territoriales, entonces la orientación dinamizadora es más clara aún. 
En función de lo anterior, resulta vital para las universidades poseer una estructura de proyectos científicos que permita responder a las prioridades nacionales, provinciales, municipales y empresariales en la mayor medida posible y en arreglo a sus restricciones de recursos financieros y humanos. En esa dirección las universidades deben disponer de una estructura óptima de proyectos científicos, entendida como la articulación y orden de las temáticas investigativas que minimicen las desviaciones entre la contribución esperada de los resultados científicos a la dinamización económica local y la contribución real que se realiza. El contar con dicha estructura no constituye la garantía absoluta de que los resultados científicos impacten en los económicos, pero constituye el paso primario en la alineación de los objetivos científicos con los económicos.

Existe un consenso amplio en la literatura consultada acerca de la enorme relevancia que tiene la ciencia, la tecnología y la innovación en las universidades para contribuir a la dinamización económica local. Para el aprovechamiento de los resultados científicos, no basta con su generación, sino que deben establecerse canales efectivos entre sus fuentes y sus destinos finales, además de que se precisan conocimientos para operar sobre el conocimiento. Por ello, no alcanza con producir ciencia, sino que las empresas, como consumidores de la nueva tecnología, deben estar preparadas para afrontar los nuevos retos.

\section{3. ¿Es necesario evaluar la contribución de la ciencia universitaria a la dinamización económica local?}

La evaluación, acción y efecto de evaluar, es la habilidad humana referida a estimar, calcular y apreciar el valor de algo (Real Academia Española, 2014). Desde esa perspectiva evaluar presupone comparar el estado de algo con una norma que sirve como medida de referencia de lo que la sociedad asume como deseable. En el caso específico de las actividades de ciencia, tecnología e innovación, es menester identificar primero esa línea de deseo para conocer luego a qué distancia se encuentra la situación observada.

La ciencia es mucho más que un conjunto de conocimientos o teorías acumuladas por el saber de la humanidad en su desarrollo como especie. Los factores intervinientes en la investigación desbordan con creces el método científico, por lo que la ciencia debe ser concebida necesariamente como una actividad social que interactúa consecuentemente con las restantes formas de actividad social, y posee un cúmulo de funciones en la sociedad, entre las que destacan: producir conocimientos, transferirlos, difundirlos y aplicarlos. La ciencia es una actividad que reclama un marco institucional apropiado dado que es un tipo específico de institución social, que necesita invariablemente el establecimiento y correcto funcionamiento de relaciones entre los diversos actores involucrados, relaciones que abarcan aspectos esencialmente de conocimiento e información, pero también éticos, políticos, jurídicos y de otros órdenes (Núñez, 1999).

Por otro lado, la tecnología es también una práctica o proceso social (Pacey, 1990), que incluye aspectos técnicos (conocimientos, destrezas, herramientas, máquinas,) pero también aspectos organizativos, muy importantes para su creación y funcionamiento. Igualmente, la innovación tecnológica como forma de interacción social, es posible y cobra sentido en dependencia de los contextos sociales. Las trayectorias tecnológicas y de innovación, que no dependen exclusivamente del impulso científico ni del tirón del mercado (aunque suelen ser buenos catalizadores), constituyen procesos multidireccionales de variación y selección que dependen de contextos socialmente constituidos. Los actores que definen esas trayectorias tecnológicas no son solo los 
que producen la ciencia, ni los que la consumen, sino también aquellos actores reguladores de los sistemas científico y productivo. Una percepción negativa de la innovación o la simple indiferencia ante la misma, puede frustrar el proceso innovativo y su impacto previsible. Así, la evaluación del impacto de la ciencia y la tecnología constituye una necesidad estratégica para constatar el desarrollo de un país, de su política científica y de su gestión en función de la sociedad y de los seres humanos que conviven en ella (Hernández, Castellanos, Núñez, Sosa, Romero y Santos, 2005).

El desarrollo, la expansión y la consolidación de los sistemas de ciencia y tecnología han conllevado el surgimiento de nuevas necesidades que emergen de la sociedad y de las propias políticas científicas, y que convierten la evaluación en una herramienta clave para la asignación o distribución de los recursos materiales o financieros, la definición de nuevos incentivos y la validación de los resultados en ciertas áreas científicas en relación con las necesidades nacionales (Sanz, 2007).

Actualmente, el proceso de evaluación de programas dirigidos al fomento de la investigación se enfrentan a retos como: las dinámicas económicas e industriales diferenciadas, los altos niveles de incertidumbre, la interacción de múltiples actores, los factores y efectos intangibles (aprendizaje, vinculaciones informales, transferencia de conocimientos) y las trayectorias científicas y tecnológicas específicas, entre otros. De ahí que quienes realizan los procesos de evaluación se ven obligados a incorporar su actividad de manera más clara en los esquemas de planeación estratégica, enfocados a considerar, entre otros aspectos, la rapidez de los procesos de aprendizaje, la capacidad de construir y desarticular redes, la capacidad de las organizaciones y las empresas para reorientarse a trayectorias con mayores oportunidades, la capacidad para anticipar e incidir en la orientación del cambio tecnológico, la movilidad inter-institucional del capital humano y los consecuentes flujos de conocimiento, e incorporar nuevos actores al diseño de evaluaciones más democráticas y transparentes (Jaso, 2007).

Los sistemas de ciencia, tecnología e innovación de cada país son extremadamente complejos y heterogéneos, lo que determina que el desarrollo y la difusión de la ciencia y la tecnología sean procesos complicados y muy difíciles de cuantificar. Los resultados o beneficios de la ciencia son intangibles, multidimensionales, y prácticamente imposibles de contar en términos económicos, porque se trata de medir la producción y el aumento del conocimiento y este es un concepto intangible y acumulativo. Además, los resultados de la ciencia se revelan sólo indirectamente y, a menudo, con mucho retraso. Por eso, las actividades científicas y tecnológicas se cuantifican desde una perspectiva aproximada o estimada basada en indicadores o parámetros evaluativos. Dichos indicadores permiten, entre otras estimaciones, comparar los niveles científicos relativos alcanzados por los países y el reconocimiento de las áreas fuertes y débiles en estas esferas (Sancho, 2002).

La evaluación debe generar insumos para proponer otras formas en que el conocimiento científico y sus aplicaciones apoyen las políticas y programas de los gobiernos, así como posibilitar la detección de carencias con respecto a indicadores en las áreas de la ciencia y la tecnología que sea necesario diseñar e incluir. Para que los resultados de las evaluaciones posean una utilidad social real y su implementación sea más factible, deben de incorporar, desde su diseño, a los actores sociales para los cuales es relevante este proceso, sean estos investigadores, empresarios, funcionarios públicos, organizaciones ciudadanas o representantes de comunidades rurales organizadas (Spinak, 2001). 
La evaluación, como herramienta que permita guiar de forma analítica la planeación de las contribuciones que la ciencia, la tecnología y la innovación realizan en la dinamización económica local, debe permitir establecer la distancia que separa al "ser" del "deber ser" y con ello, conducir a la autorregulación de la conducta de los actores individuales y colectivos vinculados, de modo que lejos de entorpecer, favorezcan el logro de esa contribución. Por lo anterior, la evaluación de la contribución de la ciencia, la tecnología y la innovación universitarias a la dinamización económica local es un proceso necesario y complejo que necesita varias etapas consecutivas e interconectadas.

La primera premisa del proceso evaluativo es el establecimiento de la situación deseada, del estándar que la sociedad ha establecido como ideal, para poder detectar la brecha entre dicho estándar y la situación real. En esa dirección, el segundo momento en la evaluación es la medición de la realidad observada en contraposición a la línea de deseo. El vehículo idóneo para dicha cuantificación es un sistema de indicadores, lo que, para el caso específico de las actividades de ciencia, tecnología e innovación, ha devenido en la aparición de la cienciometría, disciplina que estudia la producción científica con el fin de medir y analizar la misma.

Los indicadores representan una medición agregada y compleja que permite describir o evaluar un fenómeno, su naturaleza, estado y evolución; y dado que la ciencia, la tecnología y la innovación son procesos sociales altamente dependientes de las acciones y conductas de los actores que conforman el contexto que rodea a los científicos, los indicadores de las actividades de ciencia, tecnología e innovación, como constructores sociales, deben medir aquellas acciones sistemáticas relacionadas con la generación, difusión, transmisión y aplicación de conocimientos científicos y tecnológicos. Un conjunto de indicadores complementarios, los indicadores bibliométricos, constituyen una de las herramientas más utilizadas para la medición del producto de la investigación científica, porque la documentación es el medio más prolífico y exitoso para la transferencia del conocimiento, conjuntamente con su transferencia oral por medio de conferencias y comunicaciones personales (Martínez y Albornoz, 1998; Macías, 2001; Rusell: 2004). A nivel internacional se ha avanzado considerablemente en la búsqueda de indicadores para medir los resultados de la ciencia y la tecnología a nivel internacional. Los primeros aparecieron en las décadas de 1950 y 1960 bajo la denominación de inversiones y gastos en Investigación, Desarrollo e Innovación (l+D+i). En la década de 1970 se incluyeron las patentes y la balanza de pagos tecnológicos. En la década posterior se les adicionaron los productos de alta tecnología, las disciplinas métricas, los recursos humanos y la innovación. Por último, en la década de 1990 se sumaron otros como la innovación mencionada en literatura científica, el soporte público a tecnologías industriales, las inversiones intangibles y los indicadores de tecnología e información y comunicaciones (González y Molina, 2009).

Los indicadores de ciencia y tecnología reconocidos expresan valores sobre dicha actividad en un país, y generalmente, son adaptados por las instituciones científicas para medir sus propios resultados internos. Los indicadores nacionales y los propios que genera cada institución científica, señalan los logros obtenidos en estos sectores; pero no reflejan si sus beneficios se incorporan a las dimensiones del desarrollo, y si son aprovechados y disfrutados por la sociedad en su conjunto. Estos indicadores permiten a los países comparar entre sí sus inversiones y producción científica, así como buscar diferencias en los años de gestión, con la finalidad de obtener información que sea útil al momento de evaluar la relevancia y cuantía de la investigación científica. Los indicadores de ciencia y tecnología también se consideran un reflejo del desarrollo de un país. En general, un país 
con valores altos en sus índices e indicadores sociales y económicos también presenta altas inversiones en estas esferas, adecuadas capacidades y recursos humanos formados, y un sector industrial que aprovecha dichas capacidades y que obtiene beneficios de la derivación de los conocimientos en productos y servicios. Los indicadores que miden el reconocimiento del trabajo científico, tanto si se hace por medio del número de artículos como mediante el número de citas y el factor de impacto acumulado por las revistas donde publican sus resultados, presentan comportamientos muy diferentes en dependencia de las áreas de conocimiento y no siempre apuntan al mismo colectivo cuando se atiende a su excelencia (Rondón, 2004; Jaso, 2007).

No obstante los avances registrados en materia de medición de los impactos de la actividad científica, la evaluación, como proceso exploratorio, sobrepasa los marcos de la cuantificación y busca las causas de la realidad observada. En otras palabras, el tercer momento en un proceso evaluativo es el análisis de los factores que inciden en la brecha existente entre lo deseado y lo real, y que se constituyen en problemas que precisan de medidas remediales. Dado que como ha sido explicado, las actividades de ciencia, tecnología e innovación, son construcciones sociales complejas, sobre esos factores actúan un grupo de actores de cuyas interacciones depende el rumbo que tomen los primeros.

Las señales que aporta la praxis de la contribución de la ciencia, la tecnología y la innovación universitarias a la dinamización económica local, demuestran que no basta con una identificación coincidente entre las autoridades universitarias y las autoridades económicas locales de las prioridades de desarrollo, sino que, sobre la relación entre los resultados científicos y los económicos, influyen factores y actores que deben ser evaluados en aras de corregir las distorsiones que hoy persisten. En suma, que la evaluación, como habilidad superior, incluye no solo la medición del objeto de investigación, sino además la identificación y análisis de sus factores y actores determinantes; así como la propuesta de cursos de acción para eliminar las barreras y potenciar los impulsos.

A pesar de que la evaluación es una necesidad para conseguir una adecuada contribución de la ciencia, la tecnología y la innovación universitarias a la dinamización económica local, aún no se ha avanzado lo suficiente en la propuesta de herramientas analíticas que permitan evaluar en qué medida dicha contribución es coherente con lo que se espera de la ciencia universitaria.

\section{4. ¿Cómo se ha evaluado la contribución de la ciencia universitaria a la dinamización económica local?}

El análisis de la bibliografía especializada permitió identificar dos grandes áreas de abordaje de la contribución de la ciencia universitaria a la dinamización económica local, las que, mediante un análisis de conglomerados, pueden clasificarse en análisis teóricos y en propuestas metodológicas y normativas.

a) Antecedentes teóricos referidos a la contribución de la ciencia universitaria a la dinamización económica local

Aunque los estudios esencialmente teóricos coinciden en identificar el rol crucial de la ciencia universitaria para el desarrollo local, poseen algunas diferencias en el reconocimiento a la manera en que esa ciencia se imbrica con el tejido productivo y permite dinamizar la economía. Los estudios más completos y reconocidos por la comunidad académica son: 
- los que analizan la contribución de las universidades a la dinamización de las economías locales a partir del papel vigorizante de la ciencia en el sistema de innovación territorial (Mowery, 1995)

- los que consideran esta cuestión a partir de la generación de empleo, la formación y superación de la fuerza de trabajo, la transferencia de tecnología, el apoyo técnico y la cooperación para el desarrollo (Goddard, 1998b)

- los que examinan el tema a partir de la investigación académica como un activo que precisa de un fuerte enfoque comercial (Etzkowitz, 2002c)

- los que indagan en esta problemática desde la formación del capital humano y la transferencia de tecnología en forma de conocimiento (Rodríguez y Villeneuve, 2000; Alburquerque, 2003; Gabilondo, 2005; Valdizán, 2006; la Organización para la Cooperación y el Desarrollo Económico OCDE, 2009; Chauca, 2009; Ministerio de Educación de España, 2011)

- los que investigan este fenómeno a partir de una triple perspectiva: primero, cooperación universitaria como actividad profesionalizada para el refuerzo institucional; segundo, cooperación en formación, investigación científica y desarrollo tecnológico integrados en los sistemas nacionales de innovación; y tercero, cooperación para el desarrollo local a través de las redes ya existentes o de la creación de otras nuevas (Sebastián, 2000)

- los que concluyen que la contribución citada parte de la necesidad de vinculación de la formación con las competencias que se requieren en el ámbito laboral y con la necesidad de establecer estrategias de formación que relacionen a la universidad con la empresa para el desarrollo de estas competencias (Yañez, 2003; Villasmil, 2005)

- los que analizan este punto como un tercer rol universitario, insistiendo en que las instituciones universitarias son hoy en día las mayores organizaciones creadoras y difusoras de conocimiento, y por tanto; de desarrollo de capital humano como factor productivo de avanzada (Mercado, 2005; Didrickson, 2006; el Centro Interuniversitario de Desarrollo, 2007; Díaz, 2009)

- los que trabajan el fenómeno a partir del protagonismo en las redes de I+D+i territoriales (Royero, 2006)

- los que estudian la cuestión a partir de la necesidad de priorizar la educación universitaria como un factor de transformación social que atiende a las necesidades de su entorno, actúa en la construcción de una sociedad económicamente más desarrollada e influye en los modelos y orientaciones del desarrollo económico, industrial, social y cultural de su región (Martínez, 2006b)

- los que investigan la temática a partir de la universalización de la educación superior expresada en las funciones sustantivas de formación del profesional e investigación (Estrada, 2006)

- los que abordan este tema a partir de la creación, difusión y transmisión del conocimiento; el desarrollo de capacidades, y la deliberación pública (Brunner, 2007)

- los que presuponen la relación universidad-empresa como una parte consustancial, imprescindible e inmanente al desarrollo de ambas instituciones (Alcalá, 2000; Mora, 2006; Olaskoaga y Intxaurburu, 2000; Pérez, 2005)

A pesar de la enorme relevancia teórica de estos estudios, en los que se asume que por la propia misión de las universidades es imprescindible que su producción científica sea objeto de reflexión y evaluación debido a los imperativos del desarrollo social, ninguno de ellos propone instrumentos 
analíticos de evaluación de esa contribución, no consideran explícitamente el papel de los proyectos universitarios ni de las líneas de investigación y no conciben, al menos explícitamente, el proceso de dinamización de la economía local como objeto de acción de la ciencia universitaria.

b) Antecedentes metodológicos y normativos referidos a la contribución de la ciencia universitaria a la dinamización económica local

En relación con la normalización de las estadísticas para la ciencia y la tecnología, la OCDE establece las directrices que tienden a homogeneizar, a nivel internacional, los procedimientos para la selección y recogida de datos estadísticos en estas esferas y el uso de los subsiguientes indicadores. Dicha organización ha publicado los siguientes materiales que constituyen propuestas metodológicas normalizadas:

- Manual de Frascati (1963): diseñado para la ejecución de las encuestas sobre investigación y desarrollo experimental. Se ha convertido en la única guía internacional que existe en materia de normalización para la toma de datos estadísticos con vista a medir las inversiones en I+D. Presenta las definiciones básicas de los conceptos empleados en la actividades de I+D, la distinción entre I+D y otras actividades conexas, y establece directrices, normas y métodos para el desarrollo de las encuestas que recogen los datos estadísticos; asimismo, incluye la medición de los recursos humanos y financieros dedicados a la investigación y al desarrollo experimental.

- Manual de Oslo (1992): diseñado con las definiciones y metodología para diseñar las encuestas que recogen e interpretan los datos sobre fuentes de ideas innovadoras, inversiones e impacto de la innovación, así como los obstáculos para su realización. Su objetivo fundamental es la medición de las actividades de innovación en las empresas.

- Manual TBP (1992). Diseñado con las definiciones necesarias y los procedimientos metodológicos para compilar e interpretar datos tecnológicos para las balanzas de pago de las naciones.

- Manual de Patentes (1994): diseñado para la utilización de los datos de patentes como indicadores de la actividad tecnológica. Se presentan directrices para utilizar los datos incluidos en las bases de datos de patentes suministrados por las respectivas oficinas de cada país. Su objetivo es el uso de las estadísticas de patentes para la construcción de indicadores de ciencia y tecnología. Actualmente, los manuales sobre patentes e innovación se encuentran en proceso de revisión y se estudia la posibilidad de crear nuevos manuales para productos e industrias de alta tecnología y la biotecnología.

- Manual de Canberra (1995): con el objetivo de ofrecer un soporte adecuado a la medición de los recursos humanos dedicados a actividades de ciencia y tecnología. Contempla los efectivos de personal, tanto real como potencial, dedicados a I+D, así como los volúmenes y flujos de personal. Más recientemente, la propia organización ha desarrollado instrucciones para la utilización de los indicadores métricos como indicadores de ciencia y tecnología y que cubre tanto literatura científica como patentes.

Un conjunto de instituciones elabora y publica repertorios y bases de datos con series temporales de indicadores de ciencia y tecnología, donde se recogen los datos de inversiones, personal y gastos en $I+D$, series cronológicas de datos sobre financiación pública de I+D, distribuidas por objetivos socioeconómicos y patentes concedidas. Los más importantes son: "Main Science and Technology indicators" y "Basic science and technology statistics" (ambos de la OCDE), "Research and development annual statistics" (Eurostat), "Science and engineering indicators" (National 
Science Foundation, de Estados Unidos), "Science et technologie indicateurs" (Observatoire de la Science et la Technique, de Francia) y "Manual for statistics on scientific and technological activities" (Organización de las Naciones Unidas para la Educación, la Ciencia y la Cultura, UNESCO) por solo citar los más prestigiosos.

En América Latina la Red Iberoamericana de Indicadores de Ciencia y Tecnología (RICYT), celebra anualmente un taller de ciencia y tecnología, que ha permitido no solo la presentación anual de un informe denominado "El estado de la Ciencia: principales indicadores de ciencia y tecnología iberoamericanos e interamericanos", sino que ha marcado pautas en el reconocimiento e impacto del quehacer científico de los países de la región, publicando incluso el "Manual de Bogotá", con normas y definiciones para la medición de la actividad innovadora en Iberoamérica. La publicación antes citada es el único compendio que ofrece indicadores de producción científica recogidos de una serie de prestigiosas bases de datos internacionales en temas científicos específicos, además del Science Citation Index, con el objetivo de hacer posible la realización de mejores comparaciones entre los países iberoamericanos, por medio de indicadores de producción científica. Dentro del trabajo desplegado por RICYT destaca el desarrollo de guías propias para la medición y evaluación de los sistemas nacionales de innovación de los países del área, tomando como fundamento los distintos manuales elaborados por la OCDE y la Unión Europea (UE). Sin embargo el objetivo de las mismas es examinar el estado de la ciencia, la tecnología y la innovación en los diferentes países, sin llegar a evaluar, porque no está dentro de sus objetivos, la contribución a la dinamización económica local.

Por otro lado, un conjunto notable de autores realizan estudios empíricos en los que se trazan como objetivo principal valorar los impactos de la actividad universitaria sobre la producción, la renta y el empleo imputables a la universidad dentro de su área de influencia, clasifican a estos impactos en efectos directos, los que tienen origen en las actividades económicas desde la universidad y desde las personas asociadas a ella: empleados, estudiantes y visitantes; indirectos, aquellos que se generan fuera de la universidad debido a su existencia; e inducidos, los ocasionados por las actividades directas e indirectas de la universidad, que producen incrementos de producción que se expanden por el conjunto de la economía regional (Brown y Heaney, 1997; Duch, García y Parellada, 2004; San Martín y Sanjurjo, 2005; Blaya, 2006; Garrido, 2007; Gallo, Villar, Llungo, Pardo y Mancha, 2008; Pastor y Pérez, 2008).

Las herramientas analíticas propuestas para evaluar estos impactos emplean indicadores $y$ variables interesantes para obtener mediciones en los contextos específicos en que fueron aplicados, pero no proponen procedimientos para evaluar, más allá de la simple cuantificación, la contribución de la ciencia universitaria a la dinamización económica local, ni cómo determinar la estructura óptima de proyectos científicos para potenciarla.

A pesar de la enorme relevancia que poseen los estudios citados y de constituir valiosos antecedentes, aun se constata una deuda en el estado del arte, acerca de cómo evaluar la contribución de la ciencia universitaria a la dinamización económica local. La revisión bibliográfica permite construir un robusto corpus teórico sobre la relación entre los resultados científicos universitarios y el dinamismo de la actividad económica territorial, pero deja al descubierto la ausencia de propuestas metodológicas que evalúen esa relación con procedimientos específicos. 


\section{CONSIDERACIONES FINALES}

Desarrollo es satisfacción de necesidades y calidad de vida. Desarrollo es más que crecimiento, pero no existe satisfacción de necesidades sin recursos disponibles. Para tener riqueza material es vital producir bienes y servicios, y hacerlo de manera ascendente y sostenible en el tiempo. Para producir más hay que elevar la capacidad de producción y el nivel de actividad. Por ello, aunque desarrollo es más que crecimiento, no puede concebirse sin una economía dinámica, en la que prime el crecimiento sostenido en las actividades económicas del territorio, con un impacto significativo en las principales variables de la actividad económica local y en la elevación del nivel de vida de la población.

La explicación fundamental de las diferencias en el crecimiento entre una región y otra son las instituciones que protagonizan los procesos de la sociedad. La gestión activa del conocimiento que realiza una universidad le permite liderar el tránsito de los territorios de simples enclaves productivos a entornos innovadores. Sus académicos son, generalmente, los más capacitados para realizar labores consultivas y de asesoría a los gestores de las políticas públicas. Las universidades ayudan, en cualquiera de sus modalidades docentes (pregrado y posgrado) a formar las competencias exigidas por la globalidad contemporánea, que deciden si un bien es competitivo en el convulso mercado mundial.

La contemporaneidad impone la necesidad urgente e impostergable de convertir a las universidades en matrices productivas de la ciencia, la tecnología y la innovación, además de establecer de manera coherente y dinámica las relaciones entre dichas instituciones y las empresas. Pero este anhelo es solo posible si se dispone de herramientas analíticas que permitan evaluar el estado real en que se encuentra esa relación, así como la convergencia de los objetivos de cada parte, para poder reducir la brecha entre la situación deseada y la situación observada, identificando los obstáculos existentes. El estado del arte sobre el particular aún posee limitaciones en las herramientas analíticas que permitan la evaluación de la contribución de la ciencia universitaria a la dinamización económica local, lo que motiva la necesidad de diseñar un instrumento metodológico con ese objetivo. 


\section{BIBLIOGRAFÍA}

1. Acemoglu, d. Johnson, s. y Robinson, j. 2005. "Institutions as the Fundamental Cause of LongRun Growth". American Economic Review, 95, 3.

2. Alburquerque, F. 2003. Metodología para el desarrollo económico local. Santiago de Chile: CEPAL.

3. Alcalá, m. 2000. La Vinculación Universidad-Empresa y su Relación con los Sistemas de Innovación Tecnológica. [Online].

https://www.researchgate.net/publication/251804289_LA_VINCULACION_UNIVERSIDAD-

EMPRESA_Y_SU_RELACION_CON_LOS_SISTEMAS_DE_INNOVACION_TECNOLOGICA> [Consulta: abril de 2011].

4. Arocena, J. 2002. El desarrollo local: un desafío contemporáneo. Montevideo: Editorial Taurus.

5. Blaya, i. 2006. Impacto de la contribución de las instituciones de educación superior de la comunidad valenciana en el desarrollo regional. Informe de Auto Evaluación. [Online]. <https://www.docplayer.es/10464508-Impacto-de-la-contribucion-de-las-instituciones-deeducacion-superior-de-desarrollo-regional-informe-de-auto-evaluacion-la-comunidadvalenciana-en-el.html > [Consulta: junio de 2012].

6. Brown, k. y Heaney, m. 1997. "A note on measuring the economic impact of institutions of higher education". Research in Higher Education, 38, 2.

7. Brunner, J. 2007. Mercados universitarios: los nuevos escenarios de la educación superior. Santiago de Chile: FONDECYT.

8. Buarque, S. 1999. Metodología de Planejamento do Desenvolvimento Local e Municipal Sustentável. Recife: IICA.

9. Bueno, e. 2001. Los parques científicos y tecnológicos en la sociedad del conocimiento. Presentación y análisis del Parque Científico de Madrid. [Online]. <https://www.encuentrosmultidisciplinares.org/Revistan\%BA37/Eduardo_Bueno_Campos.pdf > [Consulta: marzo de 2010].

10. Castillo, j., Borroeta, b., Bayón, m. y Cordero, e. 1994. Manual de Desarrollo Local del País Vasco. Vitoria-Gasteiz: Gobierno Vasco.

11. Centro Interuniversitario de Desarrollo. 2007. Educación superior en Iberoamérica. Santiago de Chile: CINDA.

12. Chauca, p. 2009. "Papel de la universidad pública mexicana en el desarrollo local: la importancia de las actividades de investigación". Revista Pueblos y Fronteras, núm. 6, diciembre-mayo, 2008, 1-32.

13. Díaz, a. 2009. La gestión compartida universidad-empresa en la formación del capital humano. Su relación con la promoción de la competitividad y el desarrollo sostenible. [Online]. <https://www.eumed.net/tesis-doctorales/2009/amdi/ > [Consulta: mayo de 2011].

14. Didricksson, a. 2006. Contexto global y regional de la educación superior en América Latina y el Caribe. [Online]. <https://es.scribd.com/document/95772049/Tendencias-de-la-EducacionSuperior-en-America-Latina-y-el-Caribe> [Consulta: agosto de 2011].

15. Duch, n., García, j. y Parellada, m. 2004. The economic impact of Spanish public university system. An analysis to years $1998-2004$. [Online]. <https//: www.academia.edu/1123183/The_economic_impact_of_Spanish_public_university_system._ An_analysis_to_years_1998-2004> [Consulta: noviembre de 2013]. 
16. Enríquez, a. 2001. Hacia una delimitación conceptual del desarrollo regional/local. [Online]. <https://es.scribd.com/document/143349249/Desarrollo-Regional-Local> [Consulta: agosto de 2011].

17. España. Ministerio de Educación. 2011. Contribución de las universidades al progreso socioeconómico español. Madrid: Ministerio de Educación.

18. Estados Unidos. Fundación Nacional para la Ciencia NFS. 2013. Science and engineering indicators. [Online]. <https://www.nsf.gov/statistics/seind13> [Consulta: abril de 2014].

19. Estrada, v. 2006. "La gestión del conocimiento en la nueva universidad cubana". Revista Pedagogía Universitaria, XI, 2.

20. Etzkowitz, h. 2002c. "The Triple Helix of University-Industry-Government. Implications for Policy and Evaluation", Working Paper 2002-11, ISSN 1650-3821.

21. Etzkowitz, h. y Klofsten, m. 2005. "The innovating region: toward a theory of knowledge-based regional development". R\&D Management, vol. 35, 3, 243-255.

22. Gabilondo, a. 2005. La Universidad Iberoamericana en la Sociedad del Conocimiento. I Encuentro Internacional de Rectores de Universia. [Online]. $<$ https://dialnet.unirioja.es/servlet/autor?codigo=85744> [Consulta: abril de 2011]

23. Gallo, m., Vivar, m., Llungo, j., Prado, j. y Mancha, t. 2008. El Impacto Económico de la Universidad de Jaén, un enfoque de demanda. [Online]. $<$ www10.ujaen.es/sites/default/files/users/planeuja/II\%20PEUJA\%20-\%2011\%20-

\%20Referencias\%20Bibliogr\%C3\%A1ficas.pdf> [Consulta: octubre de 2014]

24. Garrido, r. 2007. "Un estudio del impacto de la Universidad sobre la Economía Local: El corredor de Henares, España". Urbano, 10, 016.

25. Goddard, j. 1998b. El papel de la Universidad en el desarrollo regional. [Online]. <https://www.unesco.org/education/educprog/wche/principal/devnr-s.html> [Consulta: mayo de 2012]

26. González, r. 2002. "La Gestión del Desarrollo Regional en Cuba. Un enfoque desde la endogeneidad". Economía Sociedad y Territorio, III, 12.

27. González, m. y Molina, m. 2009. "La evaluación de la ciencia: revisión de sus indicadores". Contribuciones a las Ciencias Sociales, 11.

28. Hernández, h. Castellanos, a. Núñez, j. Sosa, a. Romero, b. y Santos, s. 2005. "Estrategia para la proyección del impacto". Revista Cubana de Educación Superior, 1/2005.

29. Jaso, m. 2007. "Los instrumentos del evaluador de política científica y tecnológica: hacia la construcción de metodologías adecuadas a la realidad latinoamericana". Gaceta Ide@s, 2, 28.

30. Lucas, r. 1988. "On the Mechanics of Economic Development". Journal of Monetary Economics, 22.

31. Macías, c. 2001. "Papel de de informetría y de la cienciometría y su perspectiva nacional e internacional". Acimed, 9.

32. Mankiw, n., Romer, d. y Weil, d. 1992. "A Contribution to the Empirics of Economic Growth". Quarterly Journal of Economics, 107.

33. Martínez, c. 2006b. Negociación y transferencia tecnológica en el proceso de vinculación Universidad-Sector Productivo. [Online]. <https://dialnet.unirioja.es/servlet/articulo?codigo=4250562> [Consulta: septiembre de 2011].

34. Martínez, E. y Albornoz, M. 1998. Indicadores de Ciencia y Tecnología: Estado del arte y perspectivas. Caracas: Nueva Sociedad. 
35. Méndez, e. 2005. "Desarrollo territorial y local en Cuba". Temas y Reflexiones, 2.

36. Mercado, a. 2005. "El papel de la universidad en la conformación de un modelo productivo sustentable en Venezuela". Cendes, 58.

37. Mora, e. 2006. Un análisis a las barreras y obstáculos a la cooperación Universidad-Empresa. [Online]. <https://www.madrimasd.org/informacionidi/revistas/Numero2/debate3.asp> [Consulta: diciembre de 2011]

38. Mowery, D. 1995. Universities in national innovation systems. Oxford: Oxford University Press.

39. North, D. y Thomas, R. 1973. The Rise of the Western World. Cambridge: Cambridge University Press.

40. Núñez, J. 1999. La ciencia y la tecnología como procesos sociales. Lo que la educación científica no debería olvidar. La Habana: Editorial Félix Varela.

41. Nurkse, r. 1959. "Notes on Unbalanced Growth". Oxford Economic Papers N.S., 11, 3.

42. Olaskoaga, j. y Intxaurburu, m. 2000. "Iniciativas y experiencias europeas en la configuración de espacios regionales de colaboración Universidad-Empresa". Revista de Estudios Regionales, 58.

43. Orero, J. 1993. Promoción económica local en la Comunidad Valenciana. Valencia: Instituto Valenciano de Investigaciones Económicas.

44. Organización de las Naciones Unidas para la Educación, la Ciencia y la Cultura UNESCO. 2013. Manual for statistics on scientific and technological activities. [PDF]. [Consulta: junio de 2012].

45. Organización para la Cooperación y el Desarrollo Económico OCDE. 1963. Manual de Frascati. [PDF]. [Consulta: junio de 2012].

46. Organización para la Cooperación y el Desarrollo Económico OCDE. 1977. Manual de Oslo. [PDF]. [Consulta: junio de 2012].

47. Organización para la Cooperación y el Desarrollo Económico OCDE. 1992. Proposed Standard Method of Compiling and Interpreting Technology Balance of Payments Data-TBP Manual. [PDF]. [Consulta: junio de 2012].

48. Organización para la Cooperación y el Desarrollo Económico OCDE. 1994. Using Patent Data as Science and Technology Indicators-Patent Manual. [PDF]. [Consulta: junio de 2012].

49. Organización para la Cooperación y el Desarrollo Económico OCDE. 1995. The Measurement of Human Resources Devoted to S\&T-Canberra Manual. [PDF]. [Consulta: junio de 2012].

50. Organización para la Cooperación y el Desarrollo Económico OCDE. 2002. Proposed Standard Practice for Surveys of Research and Experimental Development-Frascati Manual. [PDF]. [Consulta: junio de 2012].

51. Organización para la Cooperación y el Desarrollo Económico OCDE. 2005a. Proposed Guidelines for Collecting and Interpreting Technological Innovation Data-Oslo Manual. [PDF]. [Consulta: junio de 2012].

52. Organización para la Cooperación y el Desarrollo Económico OCDE. 2009. Educación superior en el desarrollo regional y de ciudades. [PDF]. [Consulta: junio de 2012].

53. Organización para la Cooperación y el Desarrollo Económico OCDE. 2013a. Main Science and Tecnology indicators. [PDF]. [Consulta: junio de 2012].

54. Organización para la Cooperación y el Desarrollo Económico OCDE. 2013b. Basic science and technology statistics. [PDF]. [Consulta: junio de 2012].

55. Pacey, A. 1990. La cultura de la tecnología. México: Fondo de Cultura Económica. 
56. Padilla, y., Pino, j. y Aguirre, I. 2004. Desarrollo local: evolución del concepto. [PDF]. [Consulta: febrero de 2011].

57. Pastor, j. y Pérez, f. 2008. La contribución socioeconómica de las Universidades Valencianas. Instituto Valenciano de Investigaciones Económicas. [Online]. $<w w w . i v i e . e s / d o w n l o a d s / c v / c v f p e r e z . p d f>$ [Consulta: marzo de 2013].

58. Pérez, j. 2005. La relación Universidad-Empresa, análisis de experiencias mundiales y estudio de casos cubanos. [PDF]. [Consulta: abril de 2012].

59. Real Academia Española. 2014. Diccionario de la Lengua Española. www.rae.es/obrasacademicas/diccionarios/diccionario-de-la-lengua-espanola

60. Rebelo, s. 1991. "Long-Run Policy Analysis and Long-Run Growth". Journal of Political Economy, 96.

61. Red Iberoamericana de Indicadores de Ciencia y Tecnología RICYT. 2006. El estado de la ciencia. [PDF]. [Consulta: marzo de 2013].

62. Rodríguez, f. y Villeneuve, r. 2000. Universitas et civitas. Universidad y desarrollo local. [Online]. <https://riico.net/index.php/riico/article/view/899/0> [Consulta: octubre de 2012].

63. Romer, p. 1986. "Increasing Returns and Long-run Growth". Journal of Political Economy, 94(5).

64. Rondón, I. 2004. "Indicadores del impacto de la ciencia y la tecnología en la sociedad: Reflexiones y avances". Espacios, 25, 2.

65. Royero, j. 2006. "Las redes de I+D como estrategia de uso de las TIC en las universidades de América Latina". Revista de Universidad y Sociedad del Conocimiento, 3, 2.

66. Russell, j. 2004. Obtención de indicadores bibliométricos a partir de la utilización de las herramientas tradicionales de información. [Online]. <www.eventos.bvsalud.org/INFO2004/docs/es/RussellJM.pdf> [Consulta: agosto de 2013].

67. Sancho, r. 2002. "Indicadores de los Sistemas de Ciencia, Tecnología e Innovación". Economía Industrial, 343, 1.

68. San Martin, c. y Sanjurjo, e. 2002. Impacto económico de una universidad en la economía local: aplicación al caso de la Universidad de Navarra. [Online]. <https://www.navarra.es/nr/rdonlyres/d696efd2-6aaa-4ef1-b414-

e3a27109ea67/79717/11carmensanmartin.pdf> [Consulta: diciembre de 2014].

69. Sanz, I. 2007. Evaluación de la investigación y sistema de ciencia. [Online]. <https://www.researchgate.net/publication/28099891_Evaluacion_de_la_investigacion_y_sist ema_de_ciencia> [Consulta: febrero de 2011].

70. Sapag, N. 2000. Preparación y Evaluación de Proyectos. Santiago de Chile: Mc Graw Hill.

71. Solow, r. 1956. "A Contribution to the Theory of Economic Growth". Quarterly Journal of Economics, 70.

72. Spinak, e. 2001. "Indicadores cienciométricos". Acime, 9.

73. Unión Europea. Oficina Estadística de las Comunidades Europeas Eurostat. 2013. Research and development annual statistics. [Online]. <https://www.ec.europa.eu/eurostat/statisticsexplained/index.php/R_\&_D_expenditure> [Consulta: marzo de 2015].

74. Valdizán, m. 2006. Aproximación a un modelo teórico centrado en el desarrollo local desde la universidad.

[Online]. <https://www.researchgate.net/publication/39217173_Universidad_de_Burgos_y_desarrollo_ 
local_aproximacion_a_un_modelo_teorico_centrado_en_el_desarrollo_local_desde_la_Unive rsidad_deteccion_de_actividades_199495_-_200304> [Consulta: mayo de 2011]

75. Vázquez-Barquero, A. 2000a. Desarrollo económico local y descentralización: aproximación a un marco conceptual. Santiago de Chile: CEPAL.

76. Vázquez-Barquero, A. 2000b. ¿Crecimiento endógeno o Desarrollo endógeno? Montevideo: CLAEH.

77. Villasmil, m. 2005. "El papel de la universidad en el diseño de políticas para la formación de capital humano en pro del desarrollo económico local". Visión gerencial, 4, 1.

78. Yánez, c. 2003. El capital humano y las políticas sociales en la agenda del desarrollo centrado en las personas. [Online]. <www.eumed.net/tesisdoctorales/2009/amdi/GESTION\%20COMPARTIDA\%20UNIVERSIDAD\%20EMPRESA\%20EN\%20L A\%20FORMACION\%20DEL\%20CAPITAL\%20HUMANO\%20BIBLIOGRAFIA.htm> [Consulta: agosto de 2011]. 